Revue internationale P.M.E.

Économie et gestion de la petite et moyenne entreprise

\title{
Le processus de structuration dans la petite entreprise: une étude exploratoire
}

\section{Thierry Nobre}

Volume 8, numéro 2, 1995

URI : https://id.erudit.org/iderudit/1008355ar

DOI : https://doi.org/10.7202/1008355ar

Aller au sommaire du numéro

Éditeur(s)

Presses de l’Université du Québec

ISSN

0776-5436 (imprimé)

1918-9699 (numérique)

Découvrir la revue

Citer cette note

Nobre, T. (1995). Le processus de structuration dans la petite entreprise: une étude exploratoire. Revue internationale P.M.E., 8(2), 203-238.

https://doi.org/10.7202/1008355ar
Résumé de l'article

L'objet de cet article est de présenter les résultats d'une démarche de recherche clinique portant sur la structuration de la petite entreprise et plus particulièrement sur la dimension politique de cette structuration. Après avoir justifié la méthodologie utilisée et précisé les concepts centraux, l'auteur adopte une démarche descriptive et explicative qui le conduit à considérer un mécanisme général où se confrontent des forces structurantes émanant soit du chef d'entreprise, soit des autres membres de l'entreprise. Ces constatations le poussent à présenter ensuite des prescriptions pour faciliter le processus de structuration et favoriser le développement de cette catégorie d'entreprises. 


\title{
Le processus de structuration dans la petite entreprise: une étude exploratoire
}

\author{
Thierry NOBRE* \\ Université Louis-Pasteur Strasbourg
}

MOTS CLÉS

\section{Chef d'entreprise - Équipe de direction - Petite entreprise Pouvoir - Stratégie - Structuration - Structure}

\begin{abstract}
RÉSUMÉ
L'objet de cet article est de présenter les résultats d'une démarche de recherche clinique portant sur la structuration de la petite entreprise et plus particulièrement sur la dimension politique de cette structuration. Après avoir justifié la méthodologie utilisée et précisé les concepts centraux, l'auteur adopte une démarche descriptive et explicative qui le conduit à considérer un mécanisme général où se confrontent des forces structurantes émanant soit du chef d'entreprise, soit des autres membres de l'entreprise. Ces constatations le poussent à présenter ensuite des prescriptions pour faciliter le processus de structuration et favoriser le développement de cette catégorie d'entreprises.
\end{abstract}

\begin{abstract}
The aim of this article is to present the results of a clinical research process concerning the structuration of the small-sized firm and more particularly on the political dimension of this structuration. After justifying the method used and specifying the central concepts, the author adopts a descriptive and explanatory approach that leads him to consider a general mechanism where structuring strengths proceeding either from the business manager or from the other members of the firm, are confronted. These acknowledgements lead him to present then the prescriptions to facilitate the structuration process and encourage the development of this category of firms.
\end{abstract}

* Thierry Nobre est actuellement maître de conférences en sciences de gestion à l'Université Louis-Pasteur de Strasbourg. Il participe aux travaux du LARGE (Laboratoire de recherche en gestion) en privilégiant des méthodologies de recherches cliniques axées sur le développement et le contrôle organisationnel. Il est directeur des études du DESS Management international à l'Université LouisPasteur et responsable des enseignements de contrôle de gestion dans le DESS Management et stratégie des PME de l'IAE de Strasbourg. Adresse : Université Louis-Pasteur, Faculté des sciences économiques et de gestion, Laboratoire de recherche en gestion (LARGE), 38, boulevard d'Anvers, 67000 Strasbourg, France. 


\section{RESUMEN}

El objeto de este artículo es presentar los resultados de un trámite de una investigacion clínica tratando de la estructuración de la pequeña empresa y mas particularmente de la dimensión política de esta estructuración. Después de justificar la metodología utilizada y precisar los conceptos centrales, el autor adopta un trámite descriptivo y explicativo que le lleva a considerar un mecanismo general donde se confrontan las fuerzas estructurantes procediente ya del empresario ya de los otros miembros de la empresa. Estas comprobaciones le moven a presentar después las prescripciones para facilitar el proceso de structuración y favorecer el desarollo de esta clase de empresas.

\section{Introduction}

La constitution des structures de l'entreprise a fait l'objet de nombreuses études et figure en bonne place parmi les interrogations des auteurs en sciences de gestion. La plus grande partie de ces travaux s'intéresse principalement aux grandes entreprises. En revanche, ce phénomène a rarement été étudié dans la PME et a fortiori dans la petite entreprise. Compte tenu du rôle déterminant et du poids considérable des petites entreprises (Julien et Marchesnay, 1988), il nous a paru nécessaire d'analyser le processus de structuration de cette catégorie d'entreprises afin de mieux comprendre leur fonctionnement pour accroître 1'efficacité de leur management.

Avant de procéder à la présentation des résultats, nous exposons les conditions méthodologiques dans lesquelles ces travaux de recherche ont été réalisés et nous précisons les notions centrales de notre problématique, à savoir, les notions de petite entreprise et de structuration. Nous décrivons ensuite le mécanisme général qui régit la structuration dans le cadre de la petite entreprise, tel que nous l'avons observé. Afin de mieux appréhender ce mécanisme général, nous en présentons chacune des composantes, les influences qui émanent du chef d'entreprise et celles qui proviennent des membres de l'entreprise.

La description du processus de structuration, tel qu'il nous a été possible de l'observer, nous conduit ensuite à avancer quelques propositions pour en faciliter le déroulement dans la petite entreprise.

\section{Le cadre opératoire et les concepts}

Nous présentons en premier lieu le cadre opératoire de cette recherche, afin de préciser le champ de validité de nos travaux. De même, pour définir avec exactitude notre problématique, il est nécessaire d'apporter quelques précisions 
sémantiques, afin d'éviter toute ambiguïté sur les notions de petite entreprise et de structuration.

\subsection{Le cadre opératoire}

L'exposition du cadre opératoire nécessite la présentation des deux options méthodologiques retenues pour cette recherche. En ce qui concerne le rapport à l'objet étudié, c'est l'approche de la recherche-intervention qui a été retenue. Pour le développement du travail scientifique, la méthode de l'étude de cas constitue le cadre général de la progression des travaux.

\subsubsection{La recherche-intervention}

La démarche adoptée dans cette recherche induit un rapport direct à l'objet étudié qui se traduit par une intervention directe du chercheur sur les entreprises étudiées. La relation entre le chercheur et les entreprises est appelée rechercheintervention ${ }^{1}$, ce qui indique que les travaux comportent deux composantes.

1. Le chercheur intervient concrètement sur le terrain, dans l'entreprise ou l'organisation. Il effectue une prestation de service pour l'entreprise et il est en interaction avec son objet d'étude.

2. Le chercheur travaille en laboratoire; il analyse les informations qu'il a recueillies afin d'émettre des hypothèses, d'ébaucher des modèles et de participer à la construction d'une théorie.

Dans le cadre de la recherche-intervention, ces deux composantes sont étroitement imbriquées. Le chercheur, après avoir émis des hypothèses ou ébauché un modèle, doit les valider par de nouveaux tests sur le terrain. Cela implique de fortes contraintes méthodologiques, d'une part, afin de garantir la scientificité des résultats et, d'autre part, pour assurer à l'entreprise ou à l'organisation le respect de règles déontologiques concernant notamment l'anonymat et la confidentialité. En particulier, la recherche-intervention doit comporter une phase de négociation où sont définies explicitement les finalités et les conditions de réalisation. Les finalités comprennent aussi bien la production scientifique du chercheur que la production de celui-ci pour l'entreprise ou l'organisation ${ }^{2}$.

1. Le problème de la dénomination de ce type de recherche a constitué l'une des problématique du colloque « La recherche en action et en question » de l'AFCET, Collège de systémique à l'École Centrale de Paris, le 10 mars 1989.

2. Pour un approfondissement de l'évaluation de la scientificité des pratiques expérimentales de gestion, voir Girin (1990), Savall (1985) et Moisdon (1984). 


\subsubsection{Intérêt de la recherche-intervention pour l'étude du processus de structuration}

La recherche-intervention s'avère particulièrement bien adaptée à l'étude du processus de structuration, car elle permet de prendre en compte deux contraintes majeures : la dimension diachronique du processus et la sensibilité des informations nécessaires.

- La dimension diachronique du processus de structuration. La mise en place des structures de l'entreprise est un phénomène dynamique par essence. Le chercheur doit mettre en place un système d'information qui lui permet de recueillir des informations à différentes étapes de l'évolution de l'entreprise. Ces évolutions sont d'autant plus rapides dans les petites entreprises où, l'inertie étant faible, les transformations concernant par exemple l'effectif, le domaine ou le volume d'activité peuvent évoluer très rapidement. Ce besoin de collecte d'informations en temps réel est renforcé par l'atrophie de la fonction mémoire (Lemoigne, 1973) du système d'information de ces entreprises. Le chercheur, pour éviter les déformations dues au temps et aux interprétations personnelles, doit prélever ses informations le plus tôt possible en minimisant le nombre d'intermédiaires.

- La sensibilité des informations. Les informations nécessaires à l'étude du processus de structuration concernent directement les individus. Par exemple, la mise en place de nouvelles structures organisationnelles èst décidée par certains acteurs de l'entreprise ou résulte des comportements effectifs. Dans les deux cas, le chercheur doit comprendre les mécanismes qui sont à l'origine des évolutions constatées. Il s'efforce donc de recueillir des informations qui peuvent être très personnalisées.

La recherche-intervention, par la présence sur le terrain durant une longue période et l'interaction du chercheur avec les différents acteurs, permet, d'une part, d'aborder le problème de la structuration dans sa dimension diachronique et, d'autre part, de rassembler des informations de grande qualité. L'échange entre le chercheur et l'entreprise grâce au travail réalisé pour celle-ci, dans notre cas l'établissement d'un diagnostic et l'aide méthodologique à la mise en place d'outils de gestion, permet au chercheur l'accès aux informations dont il a besoin pour sa recherche, car celles-ci garantissent la validité du travail fait pour l'entreprise.

\subsubsection{Méthodologie retenue pour l'utilisation des études de cas}

La pratique de recherche fondée sur l'étude de cas peut viser plusieurs objectifs : procurer une description d'une situation (Kidder, 1992); tester une 
théorie (Pinfield, 1986) et surtout générer une théorie (Glaser et Strauss, 1967; Gersik, 1988 ; Eisenhardt, 1989 et Bryman, 1992). Notre démarche exploratoire relève de la dernière catégorie. Afin de présenter la méthodologie utilisée, nous retenons les huit étapes proposées par Eisenhardt (1989) dans le cadre de recherches réalisées à partir d'études de cas.

Ire étape : formulation d'une question de départ. La problématique est la suivante : comment se structurent les entreprises en phase de postcréation et de prédéveloppement?

$2^{e}$ étape : sélection des cas. Compte tenu de la définition précise des entreprises visées, des partenaires institutionnels (chambres de commerce, direction régionale de l'industrie, pépinières d'entreprises) ont effectué une présélection permettant de recenser des terrains expérimentaux potentiels. Sur les 264 entreprises proposées, 108 correspondaient à la définition retenue. À partir d'un premier contact téléphonique, vingt rendez-vous ont été obtenus, ce qui a conduit à la réalisation effective de sept recherches-interventions. Ensuite le chercheur a négocié son entrée dans ces entreprises en présentant la dimension expérimentale et la finalité scientifique de la rechercheintervention.

$3^{e}$ étape: fabrication des instruments de collecte des données. Les recherches-interventions se sont déroulées en deux étapes principales. Dans un premier temps, un diagnostic dysfonctionnel a été réalisé, ensuite l'intervenantchercheur a apporté une aide méthodologique aux entreprises pour la mise en place des outils de gestion. Lors de la première phase, trois techniques de recueil d'information ont été utilisées :

1. Des entretiens: tous les membres des entreprises ont fait l'objet d'un entretien.

2. La collecte de documents : pour réaliser ce diagnostic dysfonctionnel, l'intervenant-chercheur a accédé à l'ensemble des documents internes qui lui paraissaient nécessaires.

3. L'observation directe : l'intervenant-chercheur, par sa présence d'environ une semaine pour réaliser le diagnostic dysfonctionnel et lors des séances de travail d'assistance méthodologique réalisée in situ, a pu observer les comportements effectifs des différents membres des entreprises étudiées.

Lors de la deuxième phase, les informations recensées sont constituées primo par les résultats de ces réunions et les documents qui en découlent, et secundo par les observations réalisées sur les comportements et les événements, formalisées par une prise de notes intensive. 
$4^{e}$ étape: engagement sur le terrain. L'intervenant-chercheur, pour accéder aux informations confidentielles concernant le fonctionnement intime de l'entreprise, propose explicitement et formellement un échange à l'entreprise intéressée : la réalisation d'un diagnostic et une aide méthodologique à la mise en place d'outils de gestion contre l'utilisation à des fins scientifiques de toutes les informations recueillies dans le cadre de la coopération entreprisechercheur. Alors, l'entreprise se doit d'être la plus transparente possible afin de bénéficier d'une intervention la plus efficace possible.

$5^{e}$ étape: analyse des données à l'intérieur du cas. Chaque cas fait l'objet d'un rapport présentant le diagnostic. Ce diagnostic dysfonctionnel est par la suite exposé oralement à l'ensemble des membres de l'entreprise, ce qui permet une validation par l'ensemble des acteurs concernés. Les contacts ultérieurs font l'objet de prises de notes qui servent ensuite à faire évoluer le corps d'hypothèses (voir étape 7).

$\sigma^{e}$ étape : recherche de configurations transversales aux cas. La recherche de configurations transversales est réalisée à partir de l'évolution du corps d'hypothèses présenté ci-dessous.

$7 e$ étape : élaboration des hypothèses. L'ensemble des hypothèses ont été réunies dans un corps d'hypothèses qui comprend des hypothèses descriptives, explicatives et prescriptives. Les hypothèses descriptives servent à objectiver ce qui est perçu par le chercheur. La relativité de l'observation du réel liée aux biais inhérents à la relation entre le sujet observant et l'objet observé doit être prise en compte (Watzlawick, 1988). Ce phénomène est amplifié dans les sciences sociales par une interaction plus ou moins intense. Il faut donc considérer que les observations sont au départ des hypothèses, puis accumuler et recenser les faits concrets qui permettent de valider ces hypothèses descriptives afin de leur donner un statut de réalité scientifique. Par exemple, l'hypothèse descriptive "Les chefs d'entreprises ne s'investissent pas beaucoup dans les problèmes relatifs à la structuration » sera validée à partir de la répétition d'observations concrètes comme celles-ci : un chef d'entreprise déclare : «Mon rôle est de définir de nouveaux axes, de proposer de nouvelles idées; les problèmes d'intendance, ça ne m'intéresse pas!» Le chercheur constate que dans leur emploi du temps une part très faible est consacrée aux problèmes organisationnels.

Les hypothèses explicatives servent à proposer une explication aux phénomènes observés et présentés sous forme d'hypothèses descriptives. Elles sont validées à partir de liens de causalité définis entre des faits observés.

Dans les hypothèses prescriptives, le chercheur avance des propositions relatives à des solutions. Ces prescriptions sont issues des expérimentations réalisées ou, à défaut de mise en pratique, des convictions profondes du 
chercheur. Lorsque le chercheur n'a pas eu la possibilité d'effectuer ces expérimentations, il se doit de présenter les éléments qui lui permettent d'avancer les prescriptions qu'il propose.

La construction du corps d'hypothèses a été un processus itératif et continu durant la période de recherche. Elle a nécessité un aller et retour permanent entre le travail sur le terrain et le travail de réflexion en laboratoire pendant lequel ont été imbriquées une démarche logico-déductive et une démarche empirico-inductive. La démarche empirico-inductive a consisté à élaborer le corps d'hypothèses à partir des matériaux expérimentaux. La démarche logico-déductive a conduit, à chaque nouvelle intervention-recherche, à tester le corps d'hypothèses déjà élaboré pour vérifier la validité de celui-ci et éventuellement le faire évoluer.

$8^{e}$ étape : essai de formulation théorique et d'interprétation. L'essai de formulation théorique et d'interprétation a consisté à regrouper les hypothèses qui articulent les sections 3,4 et 5 de cet article en thèmes et en sous-thèmes. Notamment, cela a conduit à définir les différentes forces s'exerçant dans le processus de structuration et les incidences de ces forces sur les modalités du processus étudié.

\subsubsection{Les interventions réalisées}

Afin de présenter notre champ expérimental, nous proposons le tableau 1 où sont exposées les différentes caractéristiques des entreprises étudiées. Globalement, les entreprises constituant notre champ d'étude ont un effectif compris entre 12 et 18 personnes. Elles ont toutes moins de cinq ans et exercent leur activité dans des secteurs très diversifiés.

\subsection{Les concepts}

Afin de positionner correctement la problématique, il nous parait important de préciser les deux notions centrales: petite entreprise et structuration.

\subsubsection{La petite entreprise}

La notion de petite entreprise s'avère très large. Le critère le plus généralement admis, à savoir un effectif inférieur à 50 personnes, conduit à retenir $50 \%$ de la population des entreprises dans le cas français (Bizaguet, 1991; Julien et Marchesnay, 1988). Ainsi, on trouvera, par exemple, dans la même catégorie l'entreprise artisanale traditionnelle et l'entreprise de haute technologie appelée à se développer. 
FIGURE 1

L'élaboration du corps d'hypothèses

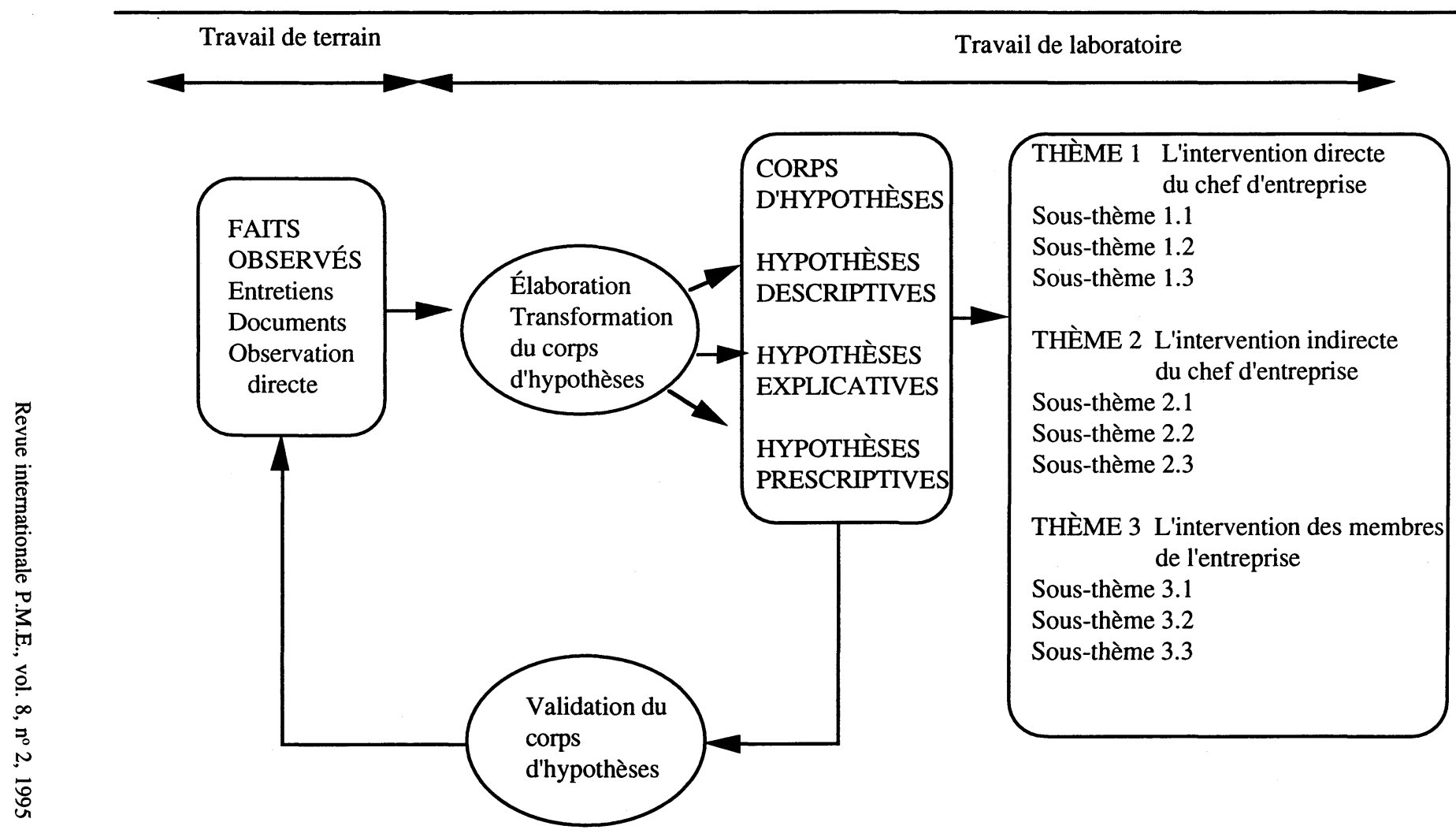


TABLEAU 1

Les entreprises étudiées

\begin{tabular}{|c|c|c|c|c|c|c|c|c|}
\hline & $\hat{\text { Âge }}$ & Effectif & Secteur & Activité & Entrepreneur & Produit & Marché & $\begin{array}{c}\text { Durée de } \\
\text { l'intervention }\end{array}$ \\
\hline $\operatorname{Cas}^{\circ} 1$ & 3 ans & 14 & $\begin{array}{l}\text { Emballage } \\
\text { plastique }\end{array}$ & $\begin{array}{l}\text { Production } \\
\text { sous- } \\
\text { traitance }\end{array}$ & Technicien & Nouveau & $\begin{array}{l}\text { Segment } \\
\text { émergent }\end{array}$ & 2 ans \\
\hline Cas $n^{\circ} 2$ & 4 ans & 15 & $\begin{array}{l}\text { Service de } \\
\text { maintenance }\end{array}$ & $\begin{array}{l}\text { Service sous } \\
\text { traitance }\end{array}$ & Technicien & $\begin{array}{c}\text { En } \\
\text { expansion }\end{array}$ & $\begin{array}{c}\text { Fortement } \\
\text { concurrentiel }\end{array}$ & 19 mois \\
\hline Cas $n^{\circ} 3$ & 4 ans & 14 & $\begin{array}{c}\text { Groupes } \\
\text { électrogènes }\end{array}$ & $\begin{array}{l}\text { Production- } \\
\text { installation } \\
\text { sous- } \\
\text { traitance }\end{array}$ & Technicien & $\begin{array}{c}\text { En } \\
\text { expansion }\end{array}$ & $\begin{array}{c}\text { Oligo- } \\
\text { polistique }\end{array}$ & 18 mois \\
\hline Cas $n^{\circ} 4$ & 4 ans & 13 & Loisir textile & Production & Commercial & Récent & $\begin{array}{c}\text { Fortement } \\
\text { concurrentiel }\end{array}$ & 16 mois \\
\hline Cas $n^{\circ} 5$ & 6 ans & 13 & $\begin{array}{l}\text { Chauffage gaz } \\
\text { pour industrie }\end{array}$ & $\begin{array}{l}\text { Production } \\
\text { installation }\end{array}$ & Technicien & Nouveau & $\begin{array}{l}\text { Fortement } \\
\text { concurrentiel }\end{array}$ & 11 mois \\
\hline Cas $n^{\circ} 6$ & 2 ans & 18 & $\begin{array}{l}\text { Agro- } \\
\text { alimentaire }\end{array}$ & Production & Gestionnaire & Maturité & $\begin{array}{c}\text { Fortement } \\
\text { concurrentiel }\end{array}$ & 1 an \\
\hline Cas $n^{\circ} 7$ & 1 an & 12 & $\begin{array}{l}\text { Ingénierie } \\
\text { financière }\end{array}$ & Service & Commercial & Nouveau & Segment émergent & 1 an \\
\hline
\end{tabular}


La définition de «petite entreprise» varie en fonction des pays et des auteurs. Cette diversité des acceptions de la notion de PE a conduit les auteurs à affiner leur définition (Julien, 1990). D'une définition monocritère et quantitative, le plus souvent ne prenant que l'effectif en compte, on est passé progressivement à une définition comprenant plusieurs critères quantitatifs et qualitatifs. Les critères quantitatifs incluent le chiffre d'affaires, le montant des actifs et la part de marché. Les critères qualitatifs relèvent d'une approche managériale et organisationnelle comprenant le type d'origine ou de propriété, la stratégie ou les objectifs de la direction, le stade de développement ou d'organisation de l'entreprise et, enfin, le secteur ou le type de marché.

Afin de tenir compte de la diversité de la réalité de la petite entreprise, voire du caractère peu opérationnel de ce concept pour l'étude des pratiques de management, et pour présenter une certaine homogénéité entre les entreprises étudiées, nous avons souhaité préciser le type de PE constituant notre champ d'étude. Il s'agit d'entreprises en phase de postcréation/prédéveloppement. Les critères qualitatifs et quantitatifs permettant de définir cette catégorie d'entreprises s'analysent suivant quatre logiques :

- dynamique: la création doit être effective, ce qui exclut certaines pratiques d'externalisation, et récente (inférieure à dix ans);

- stratégique: l'indépendance et le développement constituent deux objectifs fondamentaux;

- managériale: le chef d'entreprise est le créateur, le propriétaire, et assume effectivement les tâches de gestion;

- organisationnelle: la phase de postcréation/prédéveloppement s'amorce par la mise en place d'un troisième niveau hiérarchique qui entraîne des perturbations dans le fonctionnement de l'entreprise, puisqu'elle passe d'un mode de coordination par ajustement mutuel à un mode de coordination plus complexe et formalisé. Empiriquement, l'effectif de cette phase se situe entre 10 et 20 personnes.

La figure 2 permet de représenter cette catégorie d'entreprise.

\subsubsection{Structuration et structures}

A priori, la définition du terme «structuration» ne semble pas problématique. On peut aisément considérer qu'il s'agit du processus dynamique de la constitution des structures de l'entreprise. Pourtant, cette définition quasiment triviale est déjà lourde de présupposés, car le terme «structures » est employé au pluriel. Pour donner un sens précis au terme structuration, il nous faut donc au préalable définir ce que nous considérons comme les structures de 
FIGURE 2

La représentation des dimensions dynamique, stratégique, managériale et organisationnelle de la phase de postcréation/prédéveloppement

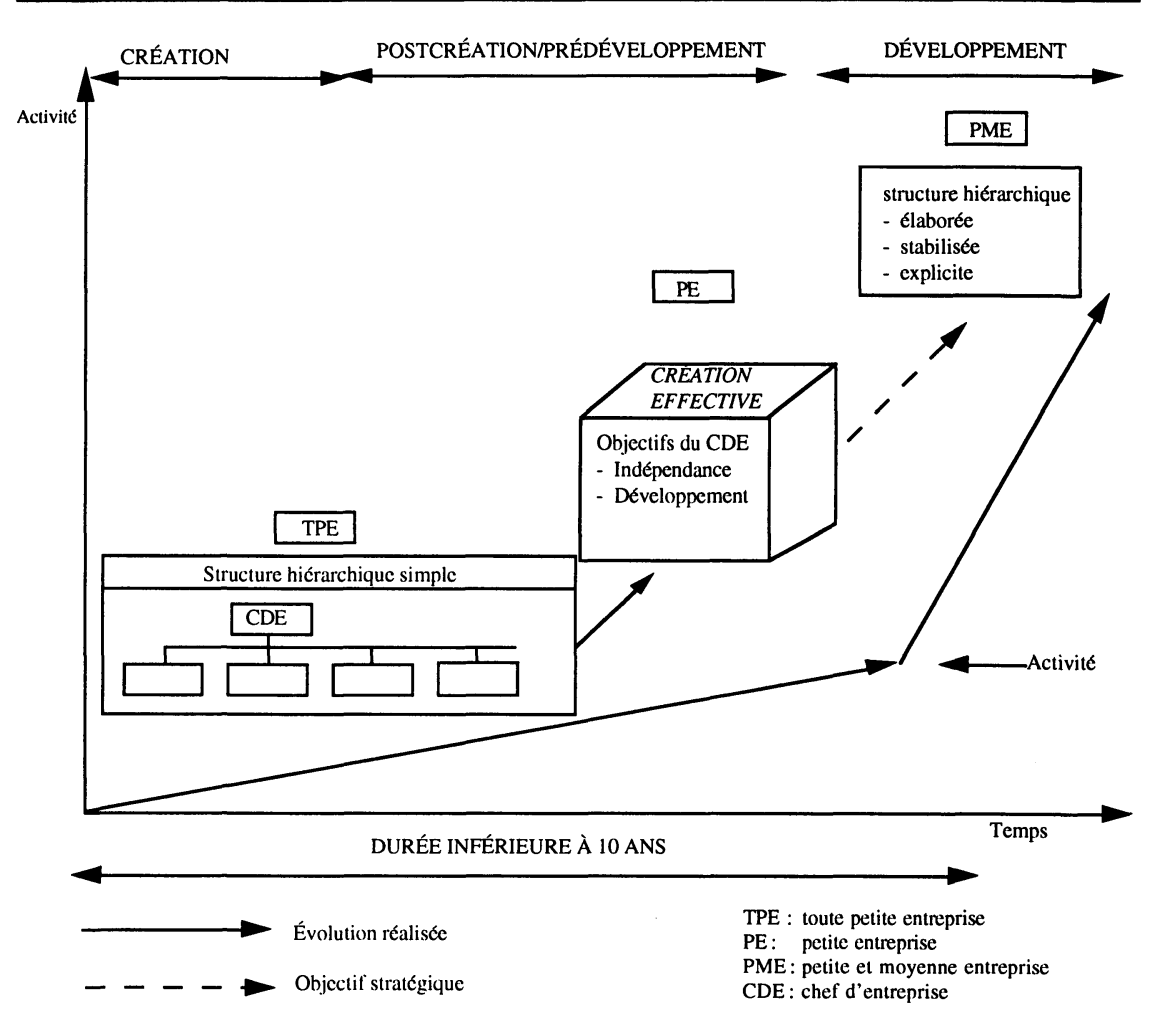

l'entreprise. Comme le rappelle Boudon (1968), le terme de structure est marqué, dans les sciences humaines, par une très forte polysémie. Cette situation se vérifie dans les sciences de gestion où les définitions de la ou des structures sont très nombreuses (Desreumaux, 1992 ; Kalika, 1984). Il nous paraît nécessaire de faire un rapide survol de la conception de différentes écoles de pensée, afin de justifier le sens que nous attribuons à ce concept.

Structures. Le concept de structure(s) nous paraît marqué par une double évolution. La première concerne la largeur du concept lui-même tandis que la seconde découle du type de démarche adoptée par les auteurs.

La largeur du concept. Dans un premier temps, on assiste au développement d'approches focalisantes. Les auteurs privilégient une catégorie d'éléments de l'entreprise. Les tenants de l'école classique considèrent les relations 
formelles; l'école des relations humaines met en avant le rôle des relations informelles; l'école néoclassique s'intéresse principalement à la direction générale. Ensuite, pour faire face à ces visions jugées trop restrictives se développent des conceptions globalisantes qui intègrent les mécanismes d'interaction entre différents éléments. Pour l'école des systèmes sociaux, la structure comprend l'ensemble des schémas de comportements qui sont stables. L'école sociotechnique privilégie l'interaction entre le système technique et le système social. L'école systémique, quant à elle, considère que la structure est l'ensemble des relations caractéristiques et stables au sein de l'entreprise.

La démarche des auteurs. Les premiers travaux conduisant à définir la structure sont fortement marqués par une démarche descriptive et normative. Les auteurs décrivent précisément ce que doit être la structure de l'entreprise. Les travaux les plus récents, par exemple, ceux réalisés par les tenants de l'école systémique, adoptent une démarche analytique et explicative. Les auteurs essaient de déterminer quels sont les éléments à l'origine du fonctionnement de l'entreprise et quelles sont les fonctions effectives remplies par ces éléments.

En nous plaçant dans la continuité de ce mouvement qui va d'une conception réduite dans le cadre d'une démarche descriptive et normative à une conception large dans le cadre d'une démarche analytique et explicative, nous retenons la définition suivante «ensemble des éléments stables et prégnants de l'organisation » développée dans l'analyse socio-économique (Savall, 1981) et rendue opérationnelle à l'aide d'une grille de cinq catégories de structures (tableau 2). La structuration de l'entreprise sera donc la constitution des éléments stables et prégnants en partant des cinq catégories de structures, soit les structures physiques, démographiques, technologiques, organisationnelles et mentales.

Structuration. L'évolution des travaux de recherche concernant l'étude des facteurs explicatifs de la structuration des entreprises comprend principalement trois étapes: les modèles retenant une cause unique, les modèles effectuant la synthèse entre les facteurs internes et les facteurs contingents et, enfin, les modèles dépassant le cadre de la double causalité de la discrétion managériale et du déterminisme contextuel.

- Les approches qui privilégient une cause unique s'articulent suivant deux logiques. La première logique, à partir d'une conception immanente privilégie, soit la stratégie (Chandler, 1962; Ansoff, 1968), soit la personnalité des dirigeants (Kets de Vries, Miller, 1982 et 1984) comme déterminants de la structuration de l'entreprise. En ce qui concerne la stratégie, le sens de la relation fait l'objet de différentes interprétations : la structure suit la stratégie (Chandler, 1962); la stratégie suit la structure (Mussche, 1974); les liaisons entre stratégie 
TABleau 2

\section{Les principaux éléments des structures d'une organisation'}

\begin{tabular}{|c|c|c|c|c|c|}
\hline Physiques & Technologiques & Organisationnelle & & Démographiques & Mentales \\
\hline $\begin{array}{l}\text { 1. Espace physique } \\
\text { 2. Configuration des } \\
\text { lieux } \\
\text { 3. Nuisances } \\
\text { - bruits } \\
\text { - chaleur } \\
\text { - toxicité } \\
\text { - éclairement }\end{array}$ & 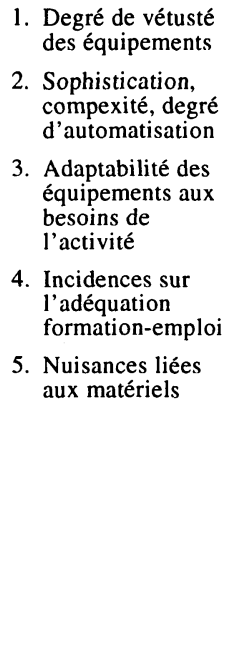 & $\begin{array}{l}\text { 1. Organigramme } \\
\text { 2. Sociogramme } \\
\text { 3. Division du } \\
\text { travail } \\
\text { 4. Méthodes } \\
\text { opératoires } \\
\text { 5. Horaires, rythmes } \\
\text { de travail } \\
\text { 6. Procédures } \\
\text { 7. Systèmes de } \\
\text { communication, } \\
\text { coordination, } \\
\text { concertation } \\
\text { 8. Système } \\
\text { d'informations } \\
\text { opérationnelles, } \\
\text { fonctionnelles }\end{array}$ & 1 & $\begin{array}{l}\text { Ajustement des } \\
\text { effectifs } \\
\text { Pyramides des } \\
\text { âges et structure } \\
\text { de la population } \\
\text { Instances de } \\
\text { représentation et } \\
\text { de concertation } \\
\text { Bassin d'emploi } \\
\text { Structures des } \\
\text { formations } \\
\text { initiales } \\
\text { Formation } \\
\text { continue } \\
\text { Structures des } \\
\text { qualifications } \\
\text { Filières } \\
\text { professionnelles } \\
\text { et } \\
\text { promotionnelles } \\
\text { Politique de } \\
\text { recrutement }\end{array}$ & $\begin{array}{l}\text { 1. Style de gestion } \\
\text { de la direction } \\
\text { 2. "Esprit-maison ", } \\
\text { culture de } \\
\text { l'organisation } \\
\text { 3. Microclimats } \\
\text { 4. Conceptions } \\
\text { sociologiques } \\
\text { culturelles } \\
\text { dominantes } \\
\text { (idéologies } \\
\text { professionnelles) }\end{array}$ \\
\hline
\end{tabular}

1. Tableau proposé par Savall et Zardet (1987).

et structures sont symétriques (Hall et Saias, 1979). La seconde logique relève d'une conception transcendantale et privilégie un facteur explicatif contextuel, l'environnement (Burns et Stalker, 1961 ; Lawrence et Lorsch, 1973), la technologie (Woodward, 1965), la taille (Pugh, Hickson et Hining, 1969).

- À l'issue de ces travaux, des recherches tentent de faire une synthèse de ces deux logiques. Ils relativisent et hiérarchisent les différentes variables (Bobitt et Ford, 1980; Khandwalla, 1977). D'autres attribuent un champ de validité à chacune des variables en fonction de la situation de l'entreprise (Montanari, 1978 ; Bourgeois, 1980; Litshert et Bonham, 1978).

- Pour dépasser la double causalité de la discrétion managériale et du déterminisme contextuel, les auteurs adoptent une démarche systémique qui débouche sur des coalignements de variables. L'environnement apparaît à la fois comme déterminant et déterminé ; la structure est aussi considérée comme le résultat d'une construction sociale ; la modification de la configuration globale résulte de variations affectant l'ensemble des variables par un processus de propagation et d'interaction (Lentz, 1981). L'approfondissement de ce schéma général conduit, à partir de l'évolution de certaines catégories 
de variables, à définir des configurations de structures, de stratégies et d'environnement (Miller et Friesen, 1980). L'interdépendance entre les différentes variables conduit à définir des «coalignements harmonieux » (Mintzberg, 1982; Capet, Causse et Meunier, 1983).

L'évolution des travaux de recherche sur le processus de structuration est marquée par trois tendances : la volonté d'aborder le problème dans toute sa complexité, le refus d'une définition trop restrictive des structures et la prise en compte de la dimension diachronique des organisations (Ranson, Hining et Greenwood, 1980; Fombrun, 1986). Ces recherches nous semblent aller dans le même sens d'une conception constructiviste ${ }^{3}$ du phénomène de la structuration (Bouchikhi, 1990 et Desreumaux, 1986).

\section{Le mécanisme général du processus de structuration des petites entreprises}

Les cas étudiés ont permis de dégager un mécanisme général comprenant deux catégories de forces découlant des comportements des acteurs au sein de l'entreprise en phase de postcréation/prédéveloppement qui conduisent à la constitution des structures de l'entreprise. Il s'agit, d'une part, des forces structurantes émanant du chef d'entreprise et, d'autre part, des forces structurantes émanant des autres membres de l'entreprise. Cette bipolarisation et cette focalisation sur le chef d'entreprise s'expliquent par le rôle central et moteur que joue celui-ci dans la petite entreprise récemment créée.

Avant de décrire dans les sections 3 et 4 comment s'exercent concrètement ces forces structurantes et quelles en sont les conséquences pour le processus de structuration, nous présentons maintenant les facteurs explicatifs des influences structurantes du chef d'entreprise et des membres de l'entreprise tels que nous avons été en mesure de les observer.

\subsection{Les forces émanant du chef d'entreprise}

Les observations réalisées permettent de retenir trois variables principales pour expliquer l'intensité des forces structurantes exercées par le chef d'entreprise. Il s'agit de la légitimité, de l'autorité et de la présence du chef d'entreprise. Comme dans la littérature, les notions de légitimité et d'autorité sont l'objet de définitions qui ne sont pas toujours convergentes, nous précisons quels sens nous retenons.

3. Pour une présentation du constructivisme, Watzlawick (1988). 


\subsubsection{La légitimité}

C'est l'aptitude à être reconnu comme le leader de l'entreprise. Cette notion de légitimité rejoint la notion définie par Weber pour qui « un pouvoir légitime est celui qui a la capacité de faire accepter ses décisions comme bien fondées, ses directives font l'objet de l'adhésion ou du moins de l'acquiescement de ceux auxquels ils sont destinés» (Boudon et Bourricaud, 19864). La légitimité détermine le potentiel de pouvoir structurant qui est accordé par les membres de l'entreprise au chef d'entreprise. Elle témoigne de la capacité de finalisation du dirigeant (Tabatoni et Jarniou, 1975 ${ }^{5}$ ), car elle permet de faire valider par les différents acteurs le projet qu'il entend développer. Nos observations nous ont permis de constater que la légitimité du chef d'entreprise n'est pas, par essence, attachée au statut de chef d'entreprise. La capacité structurante du dirigeant n'est donc pas automatiquement liée au statut de chef d'entreprise. Celui-ci doit acquérir une certaine légitimité pour avoir une influence déterminante sur la constitution des structures de son entreprise ${ }^{6}$.

\subsubsection{L'autorité}

Il s'agit de l'utilisation concrète du potentiel engendré par la légitimité. C'est la capacité d'exercer le pouvoir qui se traduit par les actions réalisées par les membres de l'entreprise sous les ordres du chef d'entreprise. En d'autres termes, c'est la capacité du chef d'entreprise d'avoir une action déterminante dans les jeux d'acteurs et d'influencer les membres de l'entreprise. Les sources de cette autorité peuvent être fort diverses (French et Raven, 1968; Crozier, 1964 ; March et Simon, 1964) mais, dans les entreprises constituant notre champ expérimental, c'est l'adéquation du modèle de référence de l'autorité du chef d'entreprise aux acteurs de l'entreprise qui est à l'origine de son pouvoir structurant.

Le rapport entre ces deux variables, légitimité et autorité, déterminant le pouvoir structurant du chef d'entreprise est régi par un effet de cliquet. Lorsque le chef d'entreprise est légitime, il peut éventuellement disposer d'une forte autorité, ce qui lui confère une influence forte sur la structuration de son entreprise. En revanche, si sa légitimité est faible, son autorité ne pourra être

4. Boudon et Bourricaud, 1986, Dictionnaire critique de la sociologie, Paris, PUF, p. 461.

5. Tabatoni et Jarniou (1975), La décomposition du système de gestion en trois soussystèmes: sous-sytème animation, sous-système organisation et sous-système finalisation.

6. Par exemple, à l'issue de la lecture du diagnostic, le chef d'une entreprise nous déclarait: «Il va falloir que je gagne mes galons de capitaine.» 
que réduite et, ainsi, son influence sur la structuration sera très fortement remise en question.

\subsubsection{La présence du chef d'entreprise}

C'est une variable beaucoup moins importante que les deux précédentes pour expliquer son influence structurante. Néanmoins, elle doit être prise en compte. La forte ou faible présence du dirigeant peut constituer un élément amplificateur ou, au contraire, modérateur qui modifie les incidences des deux précédentes variables. Une faible présence du dirigeant a tendance à atténuer l'influence structurante du dirigeant, car elle laisse une plus grande marge de manœuvre aux différents membres de l'entreprise. Ce phénomène est amplifié par le caractère rudimentaire du système d'information. Une forte présence entraîne une démultiplication de l'influence structurante, car elle déclenche un mimétisme comportemental chez les membres de l'entreprise.

La figure 3 permet de présenter les trois variables qui déterminent l'intensité de l'influence structurante du chef d'entreprise.

Les exemples étudiés conduisent, d'une part, à relativiser l'influence du dirigeant sur la constitution des structures de l'entreprise en phase de postcréation/prédéveloppement telle qu'elle a été privilégiée par les tenants du courant managérial et, d'autre part, à constater le rôle actif des membres de l'entreprise dans ce processus.

\subsection{Les forces émanant des membres de l'entreprise}

Les observations ont permis de constater que les membres de l'entreprise influencent la mise en place des structures suivant trois modalités.

- Par leur pratique professionnelle concrète et par leurs comportements effectifs, ils contribuent à la constitution des structures physiques, technologiques, organisationnelles et mentales. Leur pratique quotidienne participe, par exemple, à la définition des postes de travail, à l'élaboration de l'environnement immédiat de ces postes de travail, à l'élaboration des solutions techniques et fonctionnelles, à la définition de valeurs et de comportements de référence.

- L'activité ou la passivité des acteurs conditionne la mise en place des structures organisationnelles. Un dynamisme important ou une forte implication des membres de l'entreprise constituent un stimulus déterminant pour faire évoluer les structures organisationnelles et, plus particulièrement, le système d'information. Les acteurs face aux dysfonctionnements constatés demandent, par exemple, la mise en 
FIGURE 3

Les variables déterminant l'intensité de l'influence structurante des chefs d'entreprise

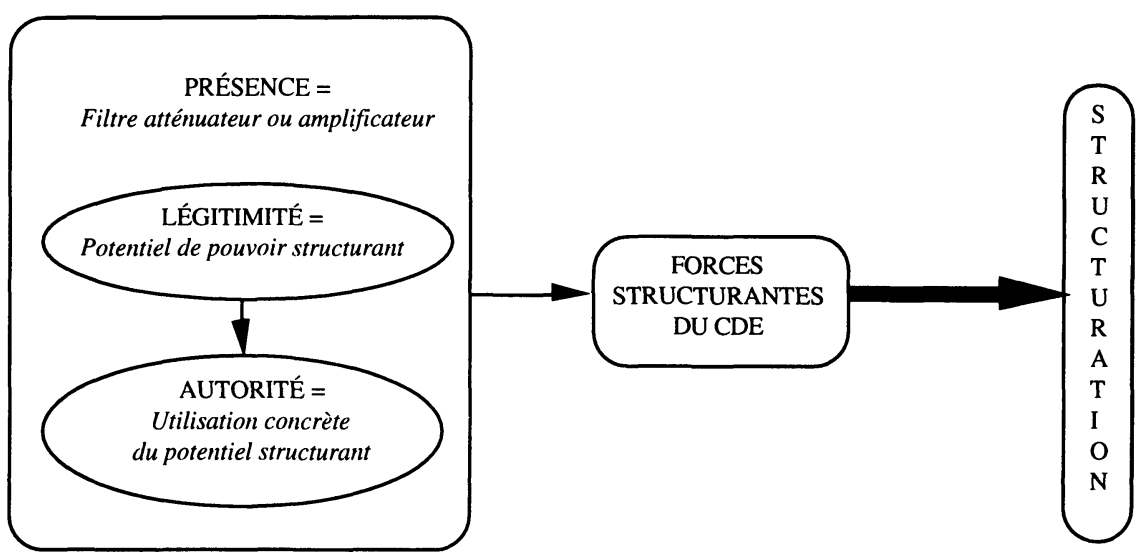

place de documents de liaison, des instructions, une définition des tâches ou des responsabilités. Au contraire, dans un contexte de forte passivité, les structures organisationnelles évoluent lentement, car elles ne bénéficient pas d'une énergie innovatrice.

- La réaction des acteurs face au comportement du chef d'entreprise contribue à la définition d'un système de valeurs propres à l'entreprise et, ainsi, à la constitution des structures mentales. Le chef d'entreprise, par la position centrale qu'il occupe, représente un véritable modèle de référence. En ne retenant dans ce modèle que certains comportements et en les adoptant, voire en les imitant ou, au contraire, en les refusant, les membres de l'entreprise jouent un véritable rôle de filtre. Ainsi, certaines valeurs illustrées par des comportements concrets sont acceptées et intériorisées ou rejetées. Par exemple, le type de gestion du temps adopté par le dirigeant entraînera, soit une conception sereine de la gestion du temps, soit un culte de «l'hyperactivité». Il en sera de même pour la conception des rapports humains dans l'entreprise, de la qualité du travail et de l'esprit d'innovation. Ainsi, lorsque les acteurs acceptent et intériorisent une grande partie des valeurs du chef d'entreprise, il se constitue une culture d'entreprise. En revanche, une forte hétérogénéité entre les valeurs du chef d'entreprise et celles qui peuvent être admises par les acteurs sera une source de dysfonctionnements, car les logiques d'actions, les langages et les raisonnements ne seront pas partagés. 
Dans les entreprises en phase de postcréation / prédéveloppement étudiées, les acteurs, par leurs comportements concrets, interviennent lors de la constitution des structures de l'entreprise. Pour expliquer les modalités de leur influence sur la mise en place des structures de l'entreprise, il nous semble important de souligner trois catégories de causes.

Le passé des acteurs. Les acteurs possèdent un modèle organisationnel de référence qu'ils ont connu au préalable. Comme dans le contexte de l'entreprise en phase de postcréation / prédéveloppement, ils ont à mettre en place des mécanismes de coordination et des relations hiérarchiques, les acteurs essaient de reproduire ce qu'ils ont connu auparavant. Il peut s'agir, par exemple, du modèle industriel avec un chef d'atelier, des chefs d'équipe et des ouvriers, ou du modèle artisanal, voire d'un modèle anti-hiérarchique. La réflexion des acteurs qui conduit à mettre en place certaines structures organisationnelles s'appuie beaucoup plus sur des références à des situations connues au préalable que sur une analyse précise de la situation concrète interne.

La stratégie des acteurs. Lors de la croissance de l'entreprise, certains salariés bénéficient d'une aspiration vers le haut de la hiérarchie. Par exemple, un ouvrier embauché à la création de l'entreprise deviendra successivement responsable de l'équipe de production puis chef d'atelier. La volonté de profiter du développement de l'entreprise pour atteindre des objectifs personnels pousse les acteurs à promouvoir tel type d'organisation qui induit une certaine catégorie de structures organisationnelles. Par exemple, pour conserver sa zone de responsabilité et pour continuer à gérer une activité de l'entreprise comme un artisan, un des membres de l'équipe de direction sera très attaché à une structure organisationnelle qui s'apparente aux structures de type divisionnel des grandes entreprises. La mise en place de structures organisationnelles par les membres de l'entreprise n'est pas un mécanisme neutre. Pour en comprendre la logique, il faut inclure dans l'analyse les objectifs individuels des principaux initiateurs.

La liberté de manœuvre des acteurs. Compte tenu de la nouveauté des problèmes qu'ils doivent résoudre et de la faible disponibilité du chef d'entreprise, les membres de l'entreprise bénéficient d'une liberté de manœuvre importante. Ils mettent en place des procédures, des modes de communication et d'information, des relations qui sont entérinées par la pratique et acquièrent le statut de structures organisationnelles, car elles se stabilisent et durent.

Les membres de l'entreprise ont ainsi une influence réelle et déterminante sur la constitution des structures des entreprises en phase de postcréation / prédéveloppement. Leurs actes concrets et leurs stratégies individuelles constituent des stimulants qui s'ajoutent à ceux provenant du chef d'entreprise. 
FIGURE 4

Mécanisme général de la structuration des entreprises en phase de postcréation / prédéveloppement

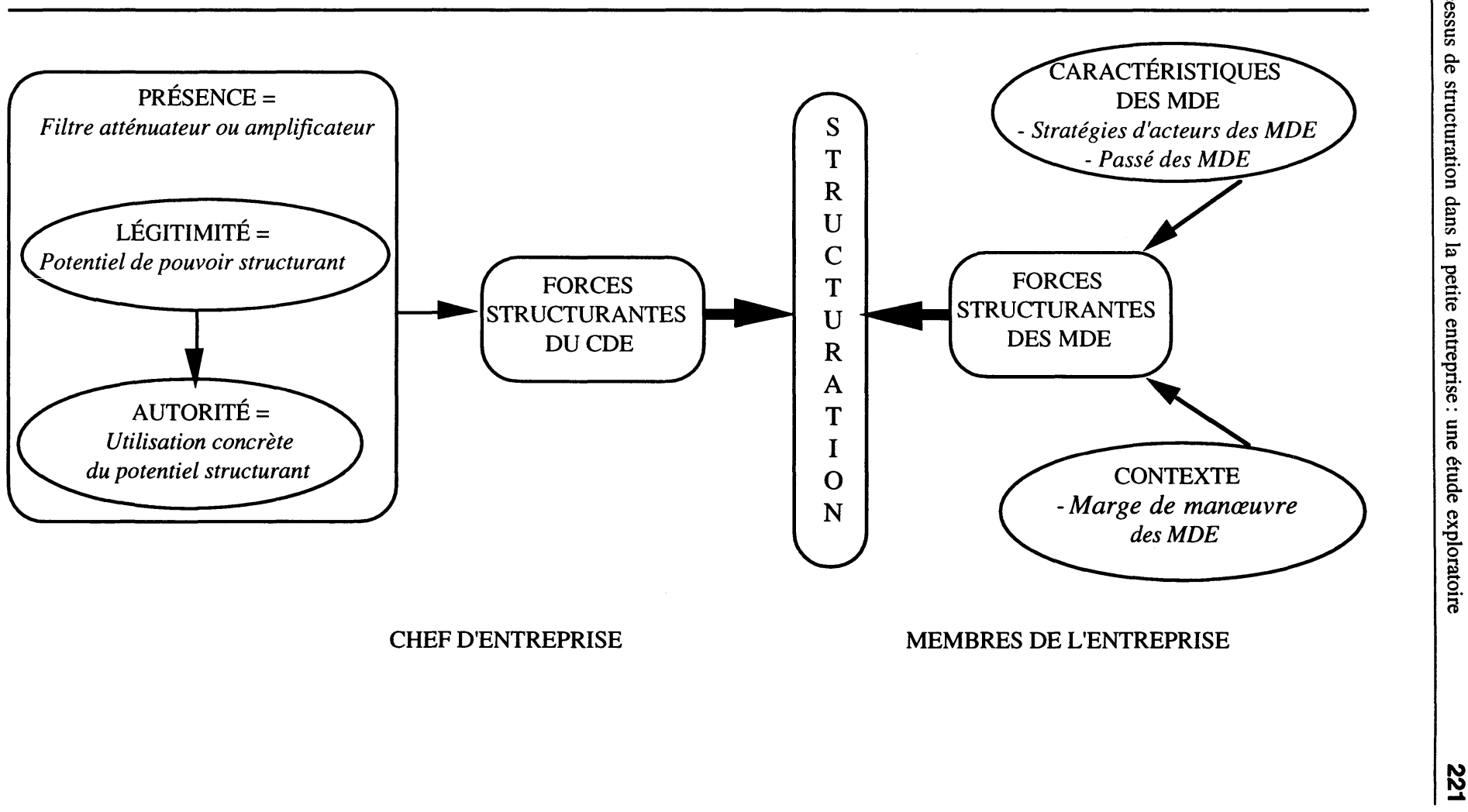


La figure 4 permet de représenter la confrontation de ces forces structurantes. Le processus de structuration de ce type d'entreprise s'apparente ainsi beaucoup plus à un mécanisme de lutte d'influences qu'à la stricte application de la volonté managériale du chef d'entreprise. Ce mécanisme s'avère ainsi éminemment politique, puisqu'il comprend la confrontation de rapports de pouvoirs entre les différents acteurs de l'entreprise. Il nous faut maintenant exposer comment ces deux catégories d'influences s'exercent concrètement.

\section{L'intervention du chef d'entreprise dans le processus de structuration}

Les observations réalisées ont conduit à considérer deux formes d'actions structurantes du chef d'entreprise: d'une part, des influences directes et, d'autre part, des influences indirectes. Les premières regroupent l'ensemble des actions réalisées par le dirigeant visant explicitement la mise en place de structures dans l'entreprise. Les secondes sont constituées par des actions dont l'objectif principal n'est pas, a priori, l'implantation de nouvelles structures mais qui, à terme plus ou moins long, ont de fortes conséquences sur le processus de structuration. Il s'agit principalement de la délégation des tâches du chef d'entreprise.

\subsection{L'intervention directe du chef d'entreprise sur le processus de structuration}

L'intervention directe du chef d'entreprise en matière de structures organisationnelles est constituée par des actions visant la mise en place de procédures, la définition de tâches, de fonctions ou de responsabilités et la constitution du système d'information de l'entreprise.

Les observations réalisées permettent de constater que les chefs d'entreprise rencontrés s'investissent peu dans ce type d'actions. Le fonctionnement interne de l'entreprise ne figure pas dans leurs principales préoccupations. Ils exercent en priorité :

- des activités commerciales, contacts avec les clients et recherche de nouveaux clients;

- des activités de recherche/développement, recherche et mise au point des nouveaux produits;

- des activités liées au financement de l'entreprise, recherche de moyens de financement de long terme ou de partenaires et gestion de trésorerie. 
Ils consacrent très peu de temps à des activités d'organisation interne et, lorsque c'est le cas, il s'agit surtout d'un travail informel. Par exemple, il n'existe aucun document ou support qui recense les décisions prises. Lors de toutes les interventions, il a été nécessaire d'instaurer la mise en place d'une procédure de comptes rendus de réunions.

Pour expliquer cette situation, quatre causes principales émergent des cas étudiés.

1. Celle qui est le plus fréquemment mise en avant par les dirigeants concerne la gestion du temps. En effet, ceux-ci ont des emplois du temps surchargés en dépit d'un nombre d'heures de travail très élevé. Bien sûr, il n'est pas question de nier cette réalité, mais elle doit être relativisée et complétée par trois autres causes.

2. Il semble patent que les chefs d'entreprise éprouvent peu d'intérêt pour des problèmes qu'ils considèrent relever de «l'intendance». Leur formation, leur cursus professionnel et leurs centres d'intérêt les poussent à se polariser sur la dimension commerciale et technique du fonctionnement de l'entreprise. Pour ce dernier aspect, nous avons été frappé par la dimension quasiment ludique qui marque la création de nouveaux produits et dans les travaux touchant à l'innovation où la notion de plaisir est loin d'être absente. Les chefs d'entreprise ont ainsi une attitude de retrait par rapport aux contingences internes du fonctionnement de l'entreprise.

3. Nous avons constaté que de graves problèmes liés à la gestion du personnel peuvent être ignorés sciemment par les dirigeants et ensuite traités trop tardivement, car ceux-ci hésitent, voire répugnent, à se heurter à la difficulté et à la complexité inhérentes aux relations humaines. Par exemple, les délais s'écoulant entre le constat de l'incapacité ou de l'inefficacité d'un responsable et la prise de décision pour faire face à cette situation s'avère très long. Or, les tâches relatives à la gestion du personnel sont éminemment structurantes. Elles ont des conséquences directes sur la constitution des structures organisationnelles, démographiques et mentales. La politique de recrutement, la définition des fonctions et des tâches, les modalités d'évaluation des performances, le type de relations existant dans l'entreprise, en partie insufflées par le dirigeant, constituent des éléments à partir desquels les structures de l'entreprise se mettent en place. C'est la méfiance des dirigeants à l'égard des problèmes de gestion du personnel qui explique cette situation. Étant implicitement conscients de la complexité des rapports humains, ils essaient d'éviter autant que possible d'avoir à y faire face. Cette volonté de jouer un 
rôle effacé dans la gestion des ressources humaines ne fait que renforcer l'inclination des chefs d'entreprise à manquer d'intérêt pour le fonctionnement interne et affaiblit ainsi leur influence sur la structuration de leur entreprise.

4. Les dirigeants ont une vision restrictive du système d'information. La comptabilité générale constitue le principal outil de référence pour répondre à leur besoin de pilotage de l'entreprise. Ils ne considèrent pas que la mise en place d'un système d'information interne dépassant le cadre comptable figure parmi leurs priorités. Par exemple, l'appréciation de l'efficacité de l'entreprise n'est mesurée qu'à partir de la connaissance du résultat net. L'entreprise doit donc attendre l'établissement des états comptables pour réagir en fonction des résultats obtenus. Cette sous-estimation de l'utilité du système d'information et de la diversité des indicateurs nécessaires concerne non seulement les informations utiles à la prise de décision du chef d'entreprise, mais aussi les activités opérationnelles. Par exemple, la circulation des informations entre les services ou les individus est très peu formalisée; cela se traduit dans la plupart des interventions par la mise en place de réseaux et de documents de liaison pour faire face aux dysfonctionnements observés. Comme les tâches conduisant à mettre en place les différentes composantes du système d'information ne sont pas considérées comme prioritaires, elles sont laissées à la libre appréciation des différents membres de l'entreprise. Compte tenu de la nature structurante de ces opérations, puisque cela entraîne, d'une part, la clarification de la répartition des tâches, des fonctions et des responsabilités et, d'autre part, la définition des flux d'informations, la conception développée par les chefs d'entreprise du système d'information tend à réduire leur influence sur le processus de structuration.

Ces quatre causes, recensées dans les entreprises analysées, sont à l'origine d'une faible intervention du dirigeant dans le fonctionnement interne. Elles entraînent une influence directe réduite de celui-ci sur le processus de structuration, compte tenu de son rôle, par ailleurs fondamental, dans ce type d'entreprise. Cela nous amène à conclure que, dans les entreprises étudiées, le processus de structuration est faiblement contrôlé de manière directe par le chef d'entreprise.

\subsection{L'intervention indirecte du chef d'entreprise sur le processus de structuration}

L'intervention indirecte sur le processus de structuration telle qu'il a été possible de l'observer résulte principalement du processus de délégation des 
tâches du chef d'entreprise. Il s'agit d'une influence indirecte, car le dirigeant, lorsqu'il délègue, ne vise pas la mise en place de structures et plus particulièrement de structures organisationnelles. C'est surtout la volonté de se débarrasser de certaines tâches qu'il ne peut plus assumer qui le pousse à cette évolution. Il s'agit beaucoup plus d'un mécanisme d'expulsion/évacuation dû à une surcharge que d'un mécanisme de construction/élaboration visant à définir un contexte de travail stable. Néanmoins, cette délégation des tâches aura des conséquences importantes en matière de formation des structures organisationnelles.

Après avoir fait le constat concernant les modalités de l'intervention indirecte du chef d'entreprise sur le processus de structuration, nous présenterons les principales raisons qui semblent à l'origine de cette évolution. Enfin, nous analyserons les conséquences de l'intervention indirecte du chef d'entreprise sur le processus de structuration en considérant le flou organisationnel qui en résulte comme la principale cause des difficultés de constitution de l'équipe de direction dans ce type d'entreprise.

\subsubsection{La répartition induite et les modalités de la délégation des tâches du chef d'entreprise}

Lorsque le chef d'entreprise délègue une partie de ce qu'il réalisait auparavant, il instaure une dynamique de répartition des tâches. Il définit implicitement le début de l'arborescence de l'organigramme en répartissant les responsabilités et les fonctions au sein de l'équipe de direction. Or, l'observation permet de constater qu'il existe un décalage important entre les aspirations du chef d'entreprise et la répartition effective. Notamment, les lignes directrices, explicites ou le plus souvent implicites, de cette répartition définie par le chef d'entreprise sont insuffisantes pour assurer un fonctionnement harmonieux et efficace de l'équipe de direction et, par conséquent, de l'entreprise.

La délégation effectuée par le dirigeant lors de la phase de postcréation/ prédéveloppement se caractérise par l'absence de définition des modalités du transfert des tâches et des responsabilités. L'apprentissage du délégataire est très fortement négligé et la transmission des procédures, lorsqu'elle est en principe réalisée, est très lapidaire. De même, il existe très rarement un système de contrôle qui aménage un transfert progressif. Bien souvent, le chef d'entreprise se débarrasse de certaines tâches sans former le délégataire et sans fixer d'objectifs. La seule formation est constituée par l'observation éventuelle que le délégataire a été en mesure de faire des méthodes utilisées précédemment par le chef d'entreprise. Ainsi, le délégataire devient-il totalement responsable de certaines tâches sans période de transition: il ne doit compter que sur lui-même. 


\subsubsection{Origine des modalités de la répartition des tâches du chef d'entreprise}

Dans les entreprises étudiées, trois éléments sont à l'origine des caractéristiques de la délégation effectivement réalisée: les contraintes de la gestion du temps, la confiance accordée par les chefs d'entreprise à leurs proches collaborateurs et l'optimisme affiché par les dirigeants.

1. Les contraintes de la gestion du temps. Comme pour l'intervention directe du chef d'entreprise sur le processus de structuration, la surcharge de travail constitue l'une des raisons pour lesquelles celuici néglige les règles et les procédures nécessaires à une délégation efficace. Il nous semble pourtant qu'elle n'est pas suffisante pour expliquer le manque d'accompagnement qui marque les opérations de délégation des tâches dans les entreprises de notre champ expérimental. C'est pourquoi nous proposons deux autres causes qui nous apparaissent déterminantes.

2. La confiance accordée par les dirigeants à leurs proches collaborateurs. Cette confiance repose principalement sur deux éléments: l'ancienneté des proches collaborateurs dans l'entreprise ou leur expérience dans une grande entreprise.

La plupart des proches collaborateurs du dirigeant sont des salariés qui sont arrivés très tôt dans l'entreprise, voire dès sa création. Le chef d'entreprise, ayant apprécié chez eux certaines qualités, leur a confié des tâches et des responsabilités supplémentaires, ce qui leur a donné le statut de responsable et de membre de l'équipe de direction.

Les autres proches collaborateurs doivent leur statut de responsable à leur expérience professionnelle au sein d'une grande entreprise. Le dirigeant les a recrutés pour bénéficier de leur savoir-faire et pour donner une dimension plus professionnelle au fonctionnement de l'entreprise.

Dans ces deux cas, il a confiance dans les compétences de ceux à qui il délègue.

3. L'optimisme des chefs d'entreprise. Lors de la délégation de leurs tâches, les dirigeants font preuve d'une forte croyance en un ajustement implicite. Ils pensent que leurs collaborateurs, malgré leur manque de préparation, arriveront toujours à trouver une solution aux problèmes qui se poseront. Cette perception de l'évolution du fonctionnement de leur entreprise émane d'une caractéristique de la personnalité des chefs d'entreprise rencontrés, à savoir un optimisme 
forcené. Il s'agit d'une attitude générale par rapport au réel; ils pensent avoir un pouvoir d'action sur celui-ci et refusent toute attitude passive. Ils font donc confiance à leurs collaborateurs, car ils pensent que ceux-ci sont suffisamment compétents pour prendre en charge des responsabilités, malgré le flou organisationnel qui accompagne cette prise en charge.

\subsubsection{L'intervention indirecte du chef d'entreprise sur le processus de structuration: du flou organisationnel aux crises de l'équipe de direction}

Les caractéristiques de la délégation des tâches développées ci-dessus semblent constituer l'un des facteurs explicatifs des fréquentes crises qui marquent la constitution des équipes de direction dans ce type d'entreprise. Six entreprises sur sept ont été l'objet de perturbations si importantes dans le fonctionnement de l'équipe de direction qu'elles pourraient aller jusqu'à remettre en cause l'existence même de l'entreprise. L'incertitude découlant du processus de délégation des tâches entraîne un flou organisationnel qui s'avère un terrain favorable au développement de conflits au sein même de l'équipe de direction.

Cette incertitude concerne les différents aspects du travail de l'équipe de direction, non seulement sa dimension fonctionnelle et opérationnelle, mais aussi sa dimension stratégique.

1. Le fonctionnement concret de l'équipe de direction est marqué par une forte incertitude en matière de répartition des responsabilités et des fonctions. De même, il existe une très forte hétérogénéité entre les méthodes de travail utilisées par différents membres de l'équipe de direction, ce qui se traduit par les difficultés constatées dans le cadre du travail en équipe. Par exemple, dans toutes les interventions, nous avons constaté une très grande inefficacité des réunions de travail regroupant l'ensemble de l'équipe de direction.

2. La composante du travail de l'équipe de direction relative à la stratégie s'avère aussi très fortement marquée par l'incertitude. On constate l'absence, au sein de l'équipe de direction, de vision commune et partagée des objectifs stratégiques de l'entreprise. Comme il n'existe pas de procédure formalisée de communication et de démultiplication de la stratégie de l'entreprise, chacun, à partir des tâches qui lui ont été déléguées par le chef d'entreprise, privilégie des axes de développement conformes à ses propres objectifs et à ce qu'il a perçu ou voulu percevoir de la stratégie de l'entreprise. 
Cette incertitude fonctionnelle et stratégique est renforcée par deux éléments : l'absence de moyens de contrôle dont dispose le chef d'entreprise et les besoins de formation de l'équipe de direction.

1. L'absence de moyens de contrôle ${ }^{7}$ mis en place par le chef d'entreprise réduit son pouvoir de régulation dès lors qu'il a délégué une tâche. Comme la délégation se fait dans l'urgence, le dirigeant ne met pas en place les mécanismes et les outils lui permettant d'effectuer des vérifications régulières pour aider son collaborateur lors du transfert de tâches, ce qui se traduit par une moindre maîtrise de l'évolution de l'entreprise.

2. Deux profils prédominants de membres de l'équipe de direction ont été observés à ce sujet. Primo, il s'avère que la réussite dans une grande entreprise n'est pas un gage suffisant de succès dans le contexte très mouvant et très passionné de l'entreprise en phase de postcréation/prédéveloppement. La primauté du savoir-faire technique s'efface devant la polyvalence, l'autonomie, la créativité et le sens des relations humaines. Secundo, lors d'une promotion professionnelle due à l'expérience et à l'ancienneté dans l'entreprise, il s'avère que les intéressés ne possèdent pas la formation nécessaire pour assumer les tâches qui leur sont confiées. Dans ces deux cas, la formation interne étant inexistante, la réussite des membres de l'équipe de direction est intimement liée à leurs qualités propres et à leur personnalité. L'incertitude créée par les conditions de réalisation de la délégation des tâches est ainsi renforcée, car l'insuffisance de formation interne fait dépendre la réussite du fonctionnement de l'équipe de direction d'éléments très subjectifs comme les qualités et les valeurs personnelles de ses propres membres.

L'incertitude découlant des modalités de réalisation effective de la délégation des tâches du chef d'entreprise permet ainsi le développement d'une forte diversité au sein de l'équipe de direction, diversité qui concerne les objectifs stratégiques perçus et visés, les méthodes de travail utilisées et les valeurs de référence. Cette diversité se traduit et se concrétise par des divergences entre les différents responsables, divergences qui ne font que renforcer les incertitudes ambiantes. Ce cercle vicieux, représenté dans la figure 5, nous apparaît comme un facteur déterminant du flou organisationnel, découlant de la délégation par évacuation/expulsion, qui provoque des conflits au sein de l'équipe de direction. Les variables qui interviennent pour déterminer la constitution des structures organisationnelles à partir de

7. Contrôle est considéré dans ses deux acceptions : vérifier et maîtriser. Voir Lauzel et Teller (1986). 


\section{FIGURE 5}

Incidences du mode de délégation des tâches du chef d'entreprise sur le mode de fonctionnement de l'équipe de direction

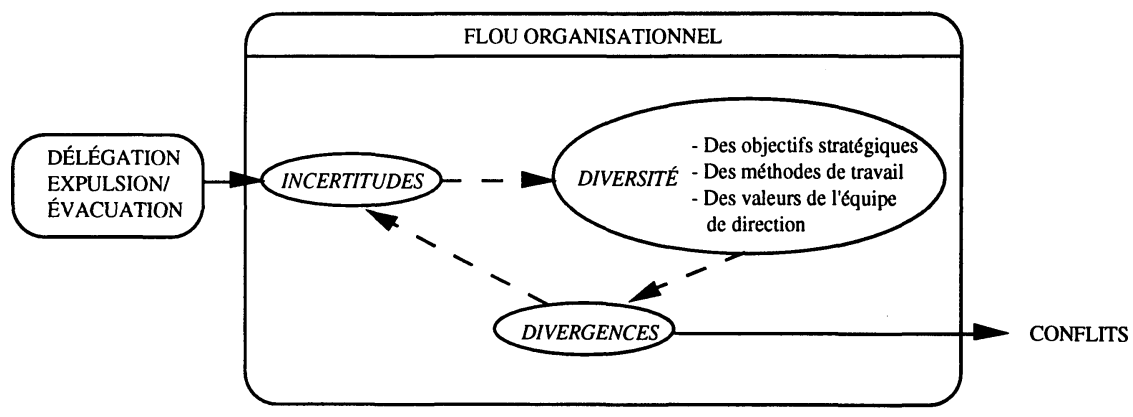

l'intervention indirecte du chef d'entreprise sont ainsi très nombreuses, puisqu'elles concernent les comportements individuels et collectifs des membres de l'équipe de direction. Les mécanismes en jeu sont complexes: ils sont relatifs aux rapports de pouvoirs qui existent entre les principaux responsables. L'influence indirecte du chef d'entreprise confère donc au processus de structuration de l'entreprise en phase de postcréation/prédéveloppement une nature très aléatoire, puisqu'elle dépend des résultats des confrontations des jeux d'acteurs.

L'étude de l'influence du chef d'entreprise sur le processus de structuration des entreprises en phase de postcréation/prédéveloppement conduit, d'une part, à constater que le contrôle exercé par celui-ci sur la mise en place des structures s'avère faible et, d'autre part, que ce processus est aléatoire, compte tenu de la nature de la délégation des tâches réalisée et du nombre de variables intervenant dans cette délégation des tâches du chef d'entreprise.

\section{L'intervention des membres de l'entreprise dans le processus de structuration}

Pour évaluer les influences des membres de l'entreprise sur le processus de structuration de l'entreprise en phase de postcréation/prédéveloppement, il est nécessaire de souligner une caractéristique essentielle du mode de fonctionnement de ce type d'entreprise: la très forte personnalisation des fonctions. Après avoir présenté ce que recouvre la forte personnalisation des fonctions, nous montrerons quelles en sont les conséquences sur le processus de structuration. 


\subsection{La forte personnalisation des fonctions}

La définition retenue pour le terme fonction est large, puisqu'elle comprend deux acceptions :

- le résultat du découpage des activités de l'entreprise : fonction commerciale, fonction de production, fonction de recherche et développement ;

- l'ensemble des tâches assumées par une personne, les fonctions de X ou Y.

Suivant la première acception, nous considérons qu'il existe une forte personnalisation des fonctions, car il est très fréquent qu'une seule personne soit responsable de 1'ensemble des tâches qui composent une fonction; il s'agit, par exemple, de la fonction commerciale (le responsable commercial est très autonome pour les contacts avec les clients, la négociation des contrats, la définition de la politique commerciale) et de la fonction recherche/développement (une seule personne assume le choix, la conception et le développement des nouveaux produits).

La deuxième acception permet la constatation suivante : certains membres de l'entreprise, notamment ceux qui sont les plus anciens et qui sont arrivés dès la création, développent un rapport très fort avec leur fonction et leur poste de travail. Ils instituent une relation qui dépasse largement le cadre de la conscience professionnelle. Dans ce cas, ces derniers considèrent que leur fonction ne peut être réalisée correctement que par eux-mêmes, ce qui s'apparente à la notion de territoire (Chevalie et Leynaud, 1983).

Dans ces deux cas de figure, la forte personnalisation des fonctions est lourde de conséquences pour la constitution des structures de l'entreprise.

\subsection{Les conséquences de la forte personnalisation des fonctions sur le processus de structuration}

La présentation des conséquences de la forte personnalisation des fonctions sur le processus de structuration nécessite l'analyse de chacune des deux acceptions retenues. Ensuite, nous nous intéresserons au mécanisme plus général du passage du mode affectif au mode professionnel dans le cadre des relations au sein de l'entreprise en phase de postcréation/prédéveloppement. Cela conduit à constater que ce mécanisme, intimement lié à la personnalisation des fonctions, influence aussi très largement le processus de structuration. 


\subsubsection{Première acception: une personne assume seule une fonction de l'entreprise}

Dans ce cas, la personnalisation des fonctions de l'entreprise constitue un risque pour la structuration, car l'efficacité de cette fonction est soumise au degré de réussite et à l'arbitraire d'une seule personne. Le danger pour le processus de structuration est imputable, d'une part, au manque de préparation et de formation de cette personne et, d'autre part, à l'absence de processus de fixation d'objectifs et de système d'évaluation des résultats. En conséquence, on assiste à une forme d'autonomie de cette fonction par rapport aux objectifs et à l'activité générale de l'entreprise, ce qui aboutit, à terme, à une désynchronisation de la fonction concernée par rapport à l'entreprise.

\subsubsection{Deuxième acception: les membres de l'entreprise s'approprient les tâches qui composent leur fonction}

Lorsque les membres de l'entreprise se sont approprié les tâches qui composent leur fonction au sein de l'entreprise, ils créent une logique d'action autonome définie en fonction d'objectifs qui leur sont propres. Ils justifient cette situation par leur ancienneté dans l'entreprise, par leur connaissance du passé ou par les moments difficiles qu'ils ont vécus aux débuts de l'entreprise. Cet état de fait constitue un obstacle à la structuration de l'entreprise car, d'une part, il entrave l'évolution des procédures internes et du système d'information et, d'autre part, il rend difficile une analyse globale des problèmes organisationnels rencontrés dans l'ensemble de l'entreprise en créant de nombreux domaines réservés.

Dans ces deux cas de figure, les différents acteurs de l'entreprise ont des comportements qui interviennent de façon active ou passive suivant des critères individuels dans la constitution progressive des structures de l'entreprise. Ces dernières sont donc constituées à partir de logiques d'action et de modèles de référence multiples, voire antagonistes.

\subsubsection{Le passage de l'affectif au professionnel}

Ces deux facettes de la forte personnalisation des fonctions entraînent une difficile évolution des relations au sein de l'entreprise vers des rapports de type professionnel, ce qui constitue un obstacle au processus de structuration. Plus particulièrement, le passage de relations très affectives, telles qu'elles existent lors de la création de l'entreprise, à des relations plus professionnalisées s'avère une transition difficile à gérer. Ces entreprises sortent d'une période où les liens tissés dépassent très largement le cadre de simples relations professionnelles. Les débuts difficiles créent en général une très forte 
solidarité entre les membres de l'entreprise et le chef d'entreprise. Le passage d'une forte convivialité initiale à une certaine impersonnalité professionnelle, causée par l'augmentation du nombre de membres de l'entreprise, déstabilise les premiers arrivants et renforce la tendance à la personnalisation des fonctions. Concrètement, cela se traduit, par exemple, par le passage difficile de l'oral à l'écrit. Cette transition est vécue comme un manque de confiance ou comme un éloignement. En effet, dans un premier temps, la totalité de la communication se fait sous forme orale et contient une forte dimension personnelle. Ensuite, pour permettre une diffusion plus large et plus efficace et pour pouvoir conserver les informations, l'entreprise doit utiliser plus largement la forme écrite. Cette transition difficile handicape la structuration de ces entreprises, car elle gêne ou empêche la formalisation et la stabilisation des procédures, des objectifs et des responsabilités. Elle permet ainsi aux comportements individuels et aux objectifs personnels de continuer de jouer un rôle essentiel dans le fonctionnement de l'entreprise.

La forte personnalisation des fonctions au sein des entreprises en phase de postcréation/prédéveloppement induit une intervention déterminante des membres de l'entreprise sur la constitution des structures et rend difficile la transition vers des rapports de type professionnel. Le rôle prépondérant joué par les stratégies d'acteurs et les objectifs individuels est à l'origine de la diversité, voire de l'antagonisme, des comportements observables. L'action des membres de l'entreprise donne au processus de structuration une nature centrifuge, car les comportements de ces derniers constituent un frein à l'évolution vers une plus grande coordination; ils mobilisent les énergies internes vers des objectifs différents et constituent un obstacle à la mise en place d'un système d'information collectif.

Le constat nous conduisant à considérer le processus de structuration comme le résultat d'une confrontation entre les influences structurantes du chef d'entreprise et celles des membres de l'entreprise étaye la conception constructiviste développée notamment par Bouchikhi et Desreumaux. L'élaboration des structures des entreprises en phase de postcréation/prédéveloppement représente un phénomène incrémental et progressif. Elle résulte de l'accumulation de micro-ajustements qui ne visent pas tous le même objectif. La constitution des structures est donc un mécanisme permanent. La nature même du processus (figure 6), faiblement contrôlé par le chef d'entreprise, aléatoire et centrifuge, s'oppose à la conception volontariste développée par le courant managérial selon lequel le chef d'entreprise gérerait et maîtriserait la structuration de son entreprise. 
FIGURE 6

Les caractéristiques du processus de structuration des entreprises en phase de postcréation / prédéveloppement

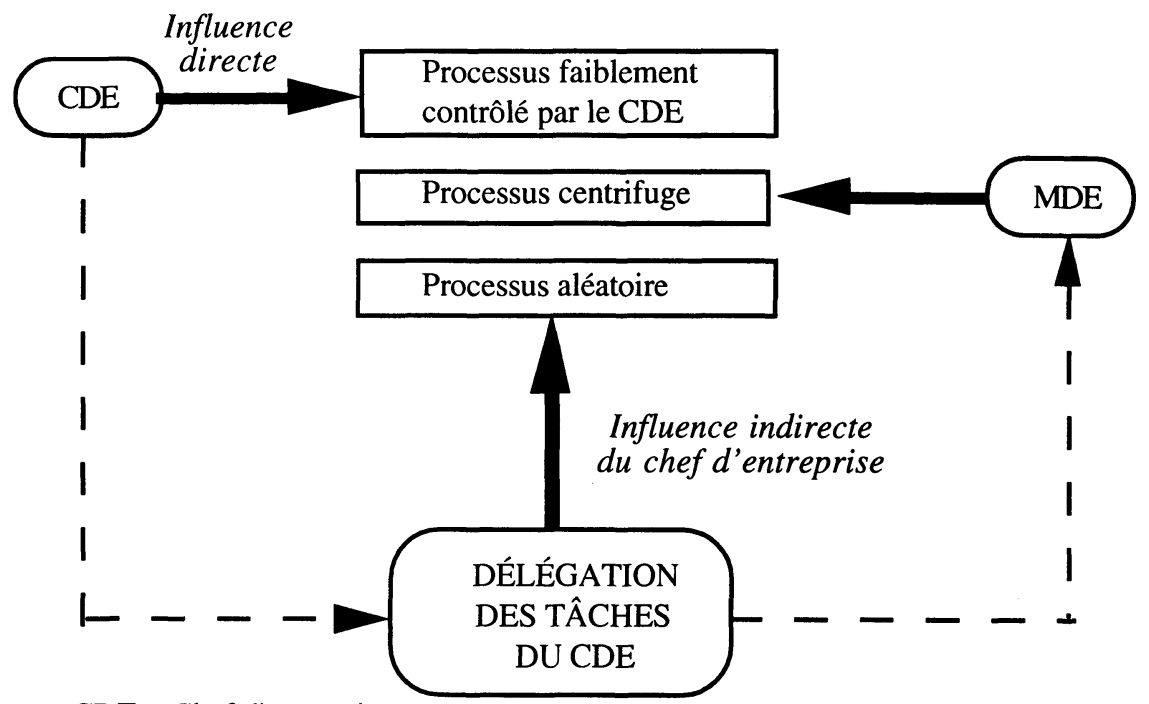

CDE : Chef d'entreprise

MDE : Membres de l'entreprise

\section{Esquisse de propositions pour faciliter la structuration de la petite entreprise}

Les difficultés rencontrées par les entreprises étudiées nous poussent à avancer quelques esquisses de propositions pour faciliter cette phase de postcréation / prédéveloppement. L'objectif principal concerne le renforcement de la cohérence du processus de structuration. Compte tenu des très fortes implications personnelles constatées, un intervenant extérieur nous paraît mieux à même de pouvoir faire le diagnostic préalable nécessaire à toute action d'envergure.

L'ensemble des actions à mener par la suite doit viser deux objectifs généraux : d'une part, renforcer l'influence effective du dirigeant sur le processus de structuration et, d'autre part, canaliser les influences des autres membres de l'entreprise. Il ne s'agit naturellement pas d'instituer un pouvoir autocratique, mais de mobiliser l'ensemble des énergies dans le même sens afin de mettre en place des structures homogènes.

Trois catégories d'actions concrètes nous semblent constituer de véritables leviers pour atteindre ces objectifs généraux. En premier lieu, le travail de l'équipe de direction doit évoluer vers un plus grand professionnalisme. Cela 
FIGURE 7

Les changements nécessaires au renforcement

de la cohérence du processus de structuration des entreprises en phase de postcréation / prédéveloppement

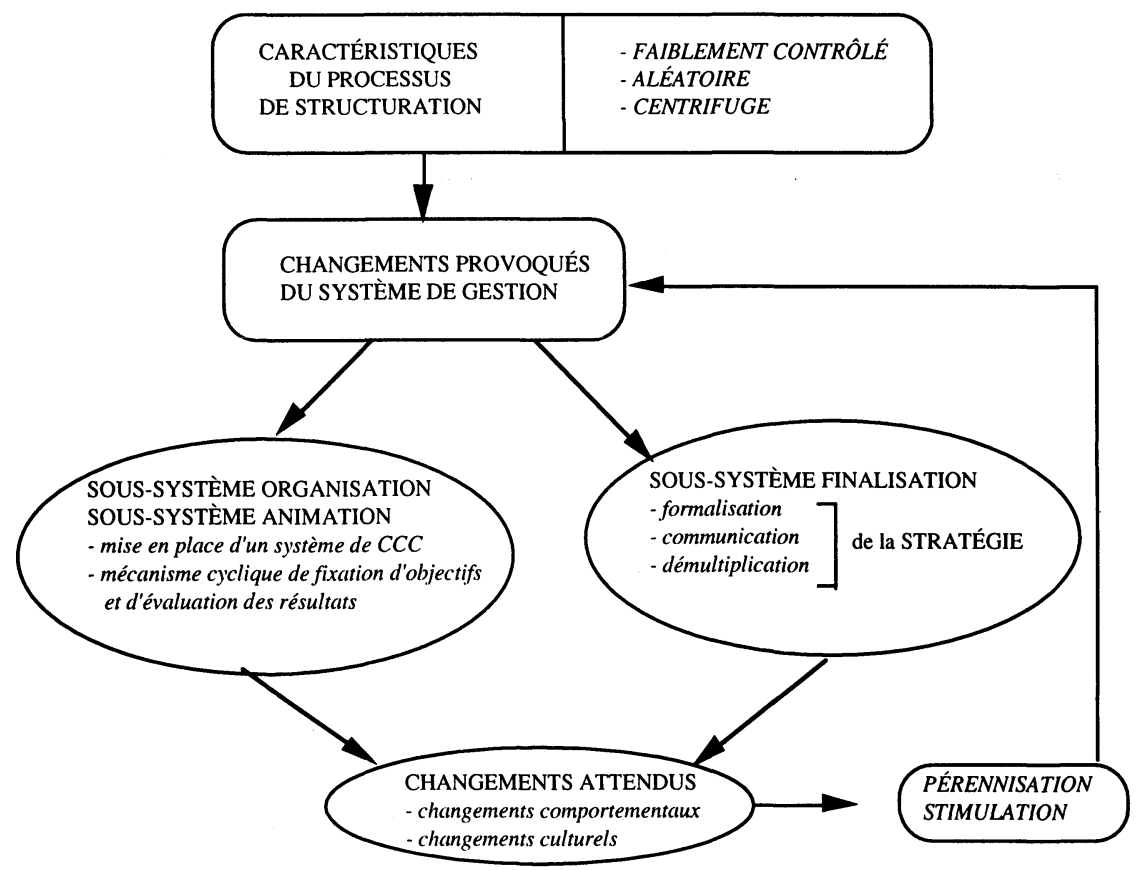

se traduit principalement par l'harmonisation des méthodes utilisées et par la mise en place d'un véritable système de communication au sein de l'équipe de direction. Ensuite, la mise en place d'un mécanisme cyclique de fixation d'objectifs et d'évaluation des résultats doit permettre un meilleur contrôle de l'évolution de l'entreprise. Enfin, une procédure de formalisation de la stratégie et une démultiplication de celle-ci en actions concrètes impliquent un accroissement de la cohérence de l'activité et du développement de ces entreprises.

Pour entreprendre ces actions concrètes, une instrumentation est nécessaire. Celle-ci comprend l'implantation d'outils de gestion et l'évolution du système d'information. Des outils de gestion comme le tableau de bord ou le plan d'actions prioritaires constituent des supports indispensables pour faire évoluer les pratiques concrètes de management. De même, l'évolution du système d'information est une étape indispensable à une évolution réelle du mode de pilotage. 
Les changements recherchés dépassent largement les seules évolutions techniques relevant de l'ingénierie du management. Il s'agit de provoquer des changements de comportements, voire des changements culturels. À partir des sous-systèmes d'organisation, d'animation et de finalisation (Tabatoni et Jarniou, 1975), l'objectif est le déclenchement de modifications qui pérennisent et stimulent l'évolution du système de gestion. Des changements concrets provoquent des transformations en profondeur dans le fonctionnement et l'évolution de l'entreprise.

\section{Conclusion}

Au terme de cette étude, il convient tout d'abord de rappeler le contexte méthodologique de ce travail. En particulier, à la richesse des informations recueillies grâce à la méthode clinique et aux interventions en profondeur réalisées, il faut opposer le nombre réduit de cas étudiés. Néanmoins, compte tenu de la diversité des entreprises étudiées, les hypothèses émises nous paraissent une base de départ acceptable pour une validation plus large à partir d'autres cas.

Le constat concernant le processus de structuration dans la petite entreprise en phase de postcréation/prédéveloppement comporte principalement trois éléments.

La mise en place des structures est un processus éminemment politique dans la mesure où elle résulte d'une lutte, formelle ou informelle, d'influences structurantes émanant du chef d'entreprise et des membres de l'entreprise. C'est le résultat de cette confrontation qui détermine la structuration de ces entreprises.

Ce mécanisme de lutte d'influences permet d'analyser les caractéristiques du processus de structuration des petites entreprises étudiées. L'étude de l'influence directe du chef d'entreprise permet de constater que la structuration est un processus faiblement contrôlé par celui-ci en raison notamment du manque de temps et du faible intérêt pour les problèmes de fonctionnement interne. L'influence indirecte du chef d'entreprise, compte tenu du mode de délégation des tâches, confère au processus de structuration une nature aléatoire à cause des nombreuses variables liées aux comportements des acteurs à qui sont déléguées les tâches. L'influence des membres de l'entreprise, à partir des différentes stratégies d'acteurs, se traduit par une dynamique centrifuge pour le processus de structuration.

La nature du processus de structuration, telle que nous l'avons observée, nous conduit à privilégier la conception constructiviste de l'évolution des entreprises, notamment en raison du caractère incrémental, progressif et interactif du processus étudié. 


\section{Bibliographie}

AFCET (1989), «La recherche-action en action et en question », Colloque du Collège de systémique à l'École Centrale de Paris, 10 mars.

ANSOFF, I. (1968), Stratégie du développement de l'entreprise, Paris, Hommes et Techniques, $288 \mathrm{p}$.

BELlet, D. (1992), Métier de conseil d'entreprise et apprentissage managérial: le cas des prestations de conseil aux dirigeants de PME/PMI, Travaux de recherche 9201, Bordeaux, CREGE, 30 p.

BAMBERGER, I. (1988), «Stratégies et structures : une analyse de leurs relations dans la perspective des nouveaux développements en Théories stratégiques», Économie et Société, $\mathrm{n}^{\circ} 8$, p. 11 à 28.

Bizaguet, A. (1991), Les petites et moyennes entreprises, Paris, Presses universitaires de France, 127 p.

BOBITT, H.R. et J.D. FORD (1980), «Decision-maker choice as a determinant of organizational structure », The Academy of Management Review, vol. 5, $\mathrm{n}^{\circ} 1$, janvier, p. 13-23.

BOUCHIKHI, H. (1990), Structuration des organisations : concept constructiviste et étude de cas, Paris, Economica, 145 p.

Boudon, R. (1968), À quoi sert la notion de "structure»? Essai sur la notion de structure dans les sciences humaines, Paris, Gallimard, 249 p.

Boudon, R. et F. BourRICAUd (1986), Dictionnaire critique de la sociologie, Paris, Presses universitaires de France, 714 p.

BOURGEOIS, L.J. (1980), «Stratégie and environnement : a conceptual integration », The Academy of Management Review, vol. 5, $\mathrm{n}^{\circ} 1$, janvier, p. 15-29.

BURnS, T. et G.M. STALKer (1961), The Management of Innovation, Londres, Tavistock, $320 \mathrm{p}$.

CAPET, M., G. CAUSSE et J. MEUNIER (1983), Diagnostic, organisation, planification d'entreprise, Paris, Economica, 587 p.

Chandler, A. (1962), Stratégies et structures de l'entreprise, Paris, Les Éditions d'Organisation, $552 \mathrm{p}$.

Chevalie, G. et A. Leynaud (1983), "Stratégie et structure de l'entreprise moyenne », Revue Française de Gestion, sept.-oct., p. 44-73.

CrozIER, M. (1964), Le phénomène bureaucratique, Paris, Seuil, 384 p.

Desreumaux, A. (1992), Structures d'entreprises, Paris, Vuibert Gestion, 332 p.

DesReumauX, A. (1986), «Formation des structures d'entreprise, revue des travaux et quelques hypothèses », Économie et Société : Sciences de Gestion, ${ }^{\circ} 6$, juin, p. 3-41. 
FOMBRUN, J.(1986), «Structural dynamics within and between organizations », Administrative Science Quarterly, vol. 31, $\mathrm{n}^{\circ} 3$, septembre, p. 338-508.

FRENCH, J.R. et B. RAVEN, (1968), «The basis of social power », dans D. Cartwright et A. Zander, Groups Dynamics, New York, Harper and Row, 380 p.

GIRIN, J. (1989), «L'opportunisme méthodique dans les recherches sur la gestion des organisations », Communication à la journée d'étude, La recherche-action en action et en question, AFCET, Collège de systémique, École Centrale de Paris, 10 mars, École polytechnique, $11 \mathrm{p}$.

GIRIN, J. (1990), «L'analyse empirique des situations de gestion : éléments de théorie et de méthode», dans Épistémologie des sciences de gestion, Ouvrage collectif, Paris, Economica, 249 p.

HALl, D.J. et M.A. SaIAS (1979), «Les contraintes structurelles du processus stratégique », Revue Française de Gestion, nº 23, p 4-16.

JUlien, P.A. (1990), «Vers une typologie multicritère des PME », Revue Internationale PME, vol. 3, nos 3-4, p. 413 à 425.

JULIEN, P.A. et M. MARCHESNAY (1988), La petite entreprise : principes d'économie et de gestion, Paris, Vuibert, $300 \mathrm{p}$.

KALIKA, M. (1984), «Contribution à la connaissance de la structure organisationnelle: essai d'analyse systémique», Thèse de doctorat d'État en sciences de gestion, Bordeaux.

KetS DE VRIES, M. et D. Miller (1984), « Neurotic style and organizational pathology », Strategic Management Journal, vol. 5, n 1, p. 35-55.

Khandwalla, P.N. (1977), The Design of Organizations, New York, Hartcourt Brace Jovanivitch, $420 \mathrm{p}$.

Lauzel, P. et R. Teller (1986), Contrôle de gestion et budget, Paris, Éd. Sirey, p. $9-15$.

LAWRENCE, P.R. et J.W. LORSCH (1973), Adapter les structures de l'entreprise : intégration ou différenciation, Paris, Les Éditions d'Organisation, 223 p.

LENTZ, R.T. (1981), « Determinants of organizational performance an interdisciplinary review», Strategic Management Journal, vol. 2, n 2, p. 131-154.

LITSHERT, R.J. et T.W. BONHAM (1978), «A conceptual model of strategy formation», The Academy of Management Review, vol. 3, p. 211-219.

MARCH, J. et H.A. SIMON (1964), Les organisations: problèmes psychologiques, Paris, Dunod, 272 p.

Miller, D. et P.H. FRIESEN (1980), « Momentum and revolution in organizational adaptation », Academy of Management Journal, vol. 23, n 4, p. 591-614. 
Miller, D., M. KeTS DE VRIES et J.M. Toulouse (1982), «Top executive locus of control and its relationship to strategy making, structure and environment », Academy of Management Journal, vol. 25, p. 237-253.

MintzBerg, H. (1982), Structures et dynamiques des organisations, Paris, Les Éditions d'Organisation, $434 \mathrm{p}$.

Mintzberg, H. (1986), Le pouvoir dans les organisations, Paris, Les Éditions d'Organisation, $679 \mathrm{p}$.

MoISDON, J.C. (1984), «La recherche en gestion et intervention », Revue Française de Gestion, septembre-octobre, p. 60-73.

MONTANARI, J.R. (1978), «Managerial discretion : an expanded model of organization choice», The Academy of Management Review, vol. 5, $\mathrm{n}^{\circ} 3$, p. 231-241.

MoRIN, P. (1985), Le Management et le Pouvoir, Paris, Les Éditions d'Organisation, $179 \mathrm{p}$.

MUSSCHE, G. (1974), «Les relations entre stratégies et structures dans l'entreprise», Revue Économique, $\mathrm{n}^{\circ}$ 1, p. 31-48.

PUGH, D.S., D.J. HICKSON et C.R. HINING (1969), «The context of organisational structures », Administrative Science Quarterly, vol. 14, p. 65-91.

RANSON, S., B. HINING et R. GREENWOOD (1980), « The structuring of organizational structures », Administrative Science Quarterly, vol. 25, n 1, p. 1-17.

SaVALL, H. (1985), «Où va la recherche en gestion? Un regard sur les recherches en sciences de gestion en France, dans les années 80 », Revue Française de Gestion, septembre-décembre, p. 95-115.

SaVAlL, H. (1981), «La notion de structure dans l'analyse socio-économique des organisations », Les cahiers de recherche en gestion de l'ISEOR, série articles $\mathrm{n}^{\circ} 2$.

SAVAlL, H. et V. ZARDET (1987), Maîtriser les coûts et les performances cachés, le contrat d'activité périodiquement négociable, Paris, Economica, $351 \mathrm{p}$.

TABATONI, P. et P. JARNIOU (1975), Les systèmes de gestion; politiques et structure, Paris, Presses universitaires de France, 231 p.

WATZLAWICK, P. (1988), L'invention de la réalité : contribution au constructivisme, Paris, Seuil, 367 p.

Weber, M. (1959), Le savant et la politique, Paris, Plon, 350 p.

WoOdwARD, J. (1965), Industrial Organizations, Oxford, Oxford University Press, 350 p. 